\title{
Fluorescence Microscopic Image Three-dimensional Reconstruction Based on Space-variant Point Spread Function Yu WANG ${ }^{\mathrm{a},{ }^{*},}$, Na ZHANG ${ }^{\mathrm{b}}$ and Huan JIANG ${ }^{\mathrm{c}}$ \\ School of Computer and Information Engineering, Beijing Technology and Business University \\ Beijing 100048, P.R. China \\ awangyu@btbu.edu.cn, 'bhangna1073@163.com, ’jh29102722@126.com
}

Keywords: Fluorescence microscopic images, 3D reconstruction, Space-variant PSF, Cubic spline.

\begin{abstract}
Three-dimensional (3D) reconstruction of fluorescence microscopic images is a challenging topic in the image processing, because the imaging system is very complex, and the point spread function (PSF) continuously varies along the optical axis. Generally, the more exact the PSF is, the higher the reconstruction accuracy is. An image reconstruction method is proposed for fluorescence microscopic sample based on space-variant PSF (SV-PSF) which is generated by cubic spline theory in this paper. Firstly, key PSFs are estimated by blind deconvolution algorithm at several depths of fluorescence microscopic image stack along the optical axis. Then, other PSFs are interpolated using cubic spline theory. Finally, a 3D microscopic specimen model is reconstructed by this group of SV-PSFs. The experimental results show that the proposed method is obviously superior to the method in which space-invariant (SI) PSF is used to reconstruct the simulated and real fluorescence microscopic images.
\end{abstract}

\section{Introduction}

Computational optical sectioning is a powerful tool for reconstructing 3D fluorescence microscopic images. Because of the existence of diffraction limitation and spherical aberration (SA) during the course of optical imaging, the fluorescence image is often degraded by blur and noise. The mathematic model for image formation is given by the following equation,

$$
g=f * h+N .
$$

where $\mathrm{g}$ is the observation image, $\mathrm{f}$ is the unknown true image, $\mathrm{N}$ is additive noise, and $h$ is the blurring operator which is decided by PSF of the system.

For simplifying the computational procedure, two assumptions are almost proposed in most image restoration algorithms. One is that the process of imaging is linear. The other is that the PSF is space- invariant. These methods [1-2], however, usually perform well only on thin specimen, because in the actual situation the PSF is space-variant along the optical axis $\mathrm{z}$ [3-5]. Only on the thin sample does the PSF vary tinily. Therefore, when data obtained from thick samples is processed using these methods, artifacts often occurs. On the other hand, for estimating all PSFs in the whole object space, extensive calculation is also an intractable problem. So some approximate algorithms are proposed in which a trade-off between the computational complexity and accuracy of image reconstruction is considered. For example, Ben Hadj et al. [6] obtained SV-PSFs by a convex combination of a group of SI-PSFs. And another 
method was also proposed [7] in which the PSF was approximately regarded as a space-invariant one within a small range. So the image stack was divided into several groups, and every group was restored by a SI-PSF. But this approach is inherently stratified and the results are not continuous between two adjacent groups. In order to smooth the block effect between any two parts of the whole PSFs, Preza et al [8] introduced a strata interpolation method to calculate the PSFs which were located at the overlapping part. However the information contained only by two adjacent parts is considered, which limits the widespread use of this kind of method.

Based on the above discussion, a 3D reconstruction method of fluorescence microscopic images is proposed using cubic spline theory. Firstly, key PSFs are estimated by blind deconvolution algorithm [9] at several depths of image stack along the optical axis z. Then, other PSFs are interpolated using cubic spline theory. Finally, a 3D microscopic specimen model is reconstructed by this group of SV-PSFs. One of advantages of our method is that the interpolation result has a higher accuracy because all key PSFs' information in the whole sample space is considered. The other advantage is that the reconstruction time can be greatly reduced because only key PSFs are estimated by blind deconvolution method, compared with those methods in which all PSFs are obtained by blind deconvolution for the accuracy of PSFs. In addition, because of the use of interpolation method, more PSFs can be generated conveniently, which is helpful to smooth different PSF at the different depths and to relieve block effect in the whole PSFs. The sketch map of the proposed method is shown in the Fig. 1.

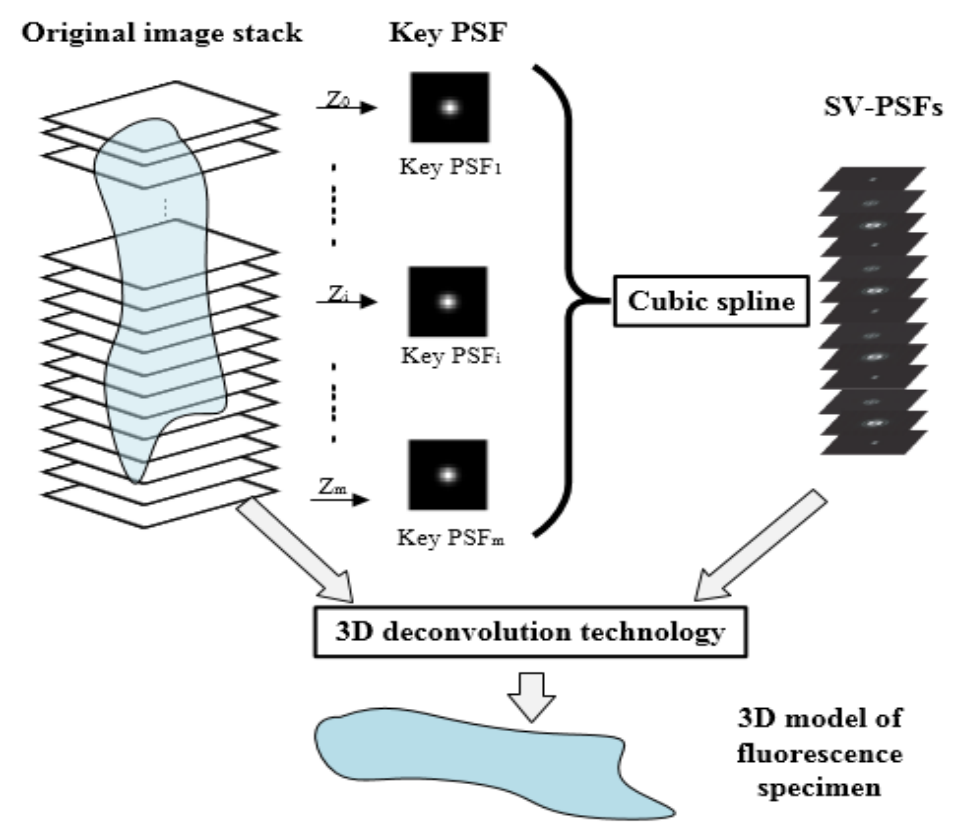

Fig.1. The sketch map of the proposed method.

\section{Methodology}

\section{Estimation of Key PSFs}

Generally, the more exact the PSF is, the higher the reconstruction accuracy is during the course of 3D reconstruction of fluorescence microscopic image. But because of the complication of the imaging system, it's too difficult to obtain all PSFs in different depth along the optical axis $\mathrm{z}$ not only by experimental measurement but also by 
theoretical model. Although blind deconvolution method [9] can accurately estimate the PSF of the degraded image, it will lead to remarkable increase of the amount of calculation if all PSFs are estimated in the whole object space. For obtaining the richer and more exact PSFs without the cost of too much the amount of calculation, key PSFs along the optical axis $z$ are firstly estimated using blind deconvolution algorithm [9]. Because 3D PSF along optical axis does not change very rapidly within a small range, key PSFs $\left\{h_{1}, h_{2}, \ldots, h_{m}\right\}$ may be calculated every few images in the image stack. In this paper key PSFs are obtained every 3 images in image stack.

\section{Generation of Other PSFs}

After obtaining a series of key PSFs, other PSFs can be generated using cubic spline theory. Regarding the key PSFs $\left\{h_{1}, h_{2}, \ldots, h_{m}\right\}$ as m interpolation nodes $n_{i}, i \in[1, m]$. At the same time the intensities of these key PSFs are defined as the node value ${ }_{i}, i \in[1, m]$, here $v_{i}$ is a two-dimensional matrix. $h_{1}$ is the initial node denoted by $\left(n_{1}, v_{1}\right)$, and $h_{m}$ is the terminal node presented by $\left(n_{m}, v_{m}\right)$. According to the theory of cubic spline, we can obtain an interpolation function with respect to the depth $\mathrm{z}$ between every two nodes. The complete form of interpolation function can be described as,

$$
S(z)=\left\{\begin{array}{cc}
S_{1}(z) & z \in\left[n_{1}, n_{2}\right] \\
S_{2}(z) & z \in\left[n_{2}, n_{3}\right] \\
\vdots & \vdots \\
S_{m-1}(z) & z \in\left[n_{m-1}, n_{m}\right]
\end{array}\right.
$$

where $S_{r}(z)(r=1,2, \ldots, m-1)$ is a three order polynomial, and can be expressed as,

$$
S_{r}(z)=a_{r} z^{3}+b_{r} z^{2}+c_{r} z+d_{r} \quad(r=1,2, \ldots, m-1) .
$$

Given the boundary condition, the polynomial coefficients $a_{r}, b_{r}, c_{r}, d_{r}$ in every

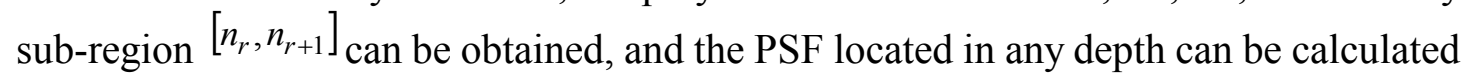
using the following equation:

$$
S_{r}\left(\Delta z_{j}\right)=a_{r}\left(\Delta z_{j}\right)^{3}+b_{r}\left(\Delta z_{j}\right)^{2}+c_{r}\left(\Delta z_{j}\right)+d_{r}, \quad\left(\Delta z_{j} \in\left[n_{r}, n_{r+1}\right], j=1,2, \ldots t\right),
$$

Where $t$ is the number of PSFs which are estimated in sub-region ' $\left[n_{r}, n_{r+1}\right]$.Therefore we can obtain PSFs at different depth in each sub-region using the interpolation function $\mathrm{S}(\mathrm{z})$. So more accurate and richer SV-PSFs in the whole fluorescence microscopic image stack can be obtained.

\section{D Reconstruction of the Specimen}

After the SV-PSFs are obtained, the fluorescence microscopic specimen can be reconstructed using these PSFs in 3D form. At present, a lot of 3D deconvolution technologies such as Richard-Lucy (RL) method [10], regularized linear least squares (LLS) method [11], linear maximum a posterior (MAP) method [12] and expectation maximization (EM) method [13] etc. can be used to reconstruct 3D fluorescence microscopic specimen. Here we choose Richard-Lucy with total variation constraint (RL-TV) [14] method to do experiments. The detailed results will be given in Section 3. 


\section{Evaluation Criteria}

Here we measure the accuracy of the interpolated PSFs using the relative squared error (RSE) [15] shown as,

$$
R S E=\frac{\|h(x)-\bar{h}(x)\|_{2}^{2}}{\|h(x)\|_{2}^{2}} .
$$

where $h(x)$ is the original PSF, and $\hat{h}(x)$ is the interpolated PSF. RSE is a positive value smaller than 1 . The smaller the RSE is, the more exactly the PSF is interpolated.

Considering that our restoration method is applied to microscopic images, the performance of preserving structure detail is of great importance. Therefore, we choose the structural similarity (SSIM) method [16] to measure the structure detail similarity between the original image $\mathrm{F}$ and the restored image $\mathrm{G}$, the equation is defined as

$$
\begin{aligned}
& \operatorname{SSIM}=l(F, G) \cdot c(F, G) \cdot s(F, G) \\
& l(F, G)=\frac{2 \mu_{F} \mu_{G}}{\mu_{F}^{2}+\mu_{G}^{2}}, \quad c(F, G)=\frac{2 \delta_{F} \delta_{G}}{\delta_{F}^{2}+\delta_{G}^{2}}, \quad s(F, G)=\frac{\delta_{F G}}{\delta_{F} \delta_{G}}
\end{aligned}
$$

where $l(F, G), c(F, G)$ and $s(F, G)$ denoted respectively the similarity information about luminance, contrast and structure between the original image and the restored one. $\mu_{F}$ and $\mu_{G}$ are respectively the mean intensity of the original image and the restored image. $\delta_{F}$ and $\delta_{G}$ are respectively the standard deviation of the original image and the restored image, and $\delta_{F G}$ is the covariance of them. SSIM is also a positive value smaller than 1 . On the contrary to RSE, the larger SSIM is, the more exactly the object is restored.

\section{Algorithmic Flow}

In this paper, a 3D reconstruction method of microscopic image stack is proposed. During the course of 3D reconstruction, it's difficult to obtain all PSFs in different depth along the optical axis z. Therefore, key PSFs are firstly estimated by means of blind deconvolution. Then other PSFs are generated using cubic spline theory. Finally, more exact 3D model of the fluorescence microscopic images can be reconstructed using all the SV-PSFs and RL-TV [14] method.

\section{Experimental Results}

For validating the efficiency of the proposed method, we compared our results with those of traditional method in terms of synthetic and real fluorescence image stack.

\section{Synthetic Data}

We firstly evaluated our method using synthetic image stack. The advantage processing synthetic image stack is that the original object and PSFs are known. Thus the quality of the restored object and estimated PSFs can be readily assessed. We can obtain the blurred and noisy image stack by convolving a group of PSFs and adding noise in every piece of image. We chose Gaussian function as the system's approximated PSF which 
has the similar shape with Bessel function. The different plane could be simulated by the Gaussian function with different variances. The form of the 3D Gaussian PSF can be described as [17],

$$
h(x, y, z)=\frac{1}{2 \pi \sigma_{z}^{2}} e^{-\frac{p^{2}+q^{2}}{2 \sigma_{z}^{2}}}
$$

where $\sigma_{z}^{2}=\sigma_{z_{0}}{ }^{2}+\alpha\left|z-z_{0}\right|, \mathrm{z}$ denotes the current plane, and $z_{0}$ denotes the central position. $\sigma_{z_{0}}^{2}$ and $\sigma_{z}^{2}$ is the variance of central position and current plane respectively. $\alpha$ is the weighting function. In this paper, we set $z_{0}=0, \alpha=0.5, \sigma_{z_{0}}^{2}=0.6, p \in[-2,2], q \in[-2,2]$, and the interval of two slices is $0.2 \mu \mathrm{m}$.

So 65 PSFs were generated using Eq. (8). Fig. 2(a) is a synthetic microscopic image stack $\left(75^{*} 100^{*} 65\right)$, and Fig. 2 (b) is a blurred and noisy image stack by convolving these generated PSFs and adding noise.

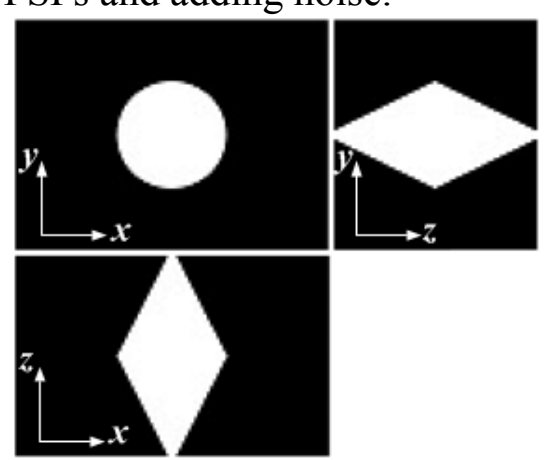

(a)

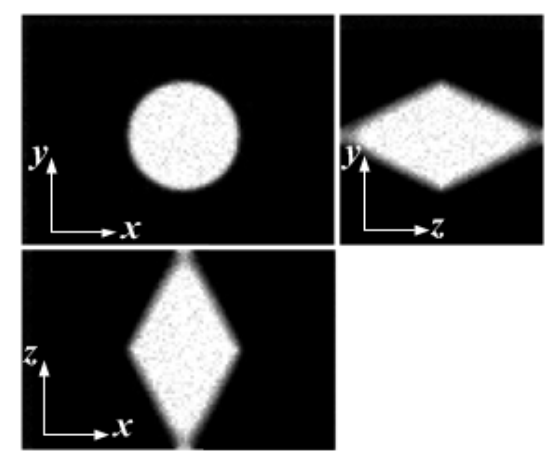

(b)

Fig.2. (a) The original image stack, and (b) the degraded image stack.

For reconstructing 3D model of synthetic microscopic image stack, 17 key PSFs were firstly estimated by blind deconvolution method [9]. Then other PSFs were interpolated using cubic spline theory (Supposing that the interpolation function meets boundary condition, i.e., ). Fig. 3(a) shows the intensities which are located in the same position of the estimated and interpolated PSFs. For proving the feasibility of the method, all PSFs in different depth were estimated using deconvolution method in advance besides the above mentioned 17 key PSFs. The errors between the estimated PSFs and the interpolated PSFs are shown in Fig. 3(b).

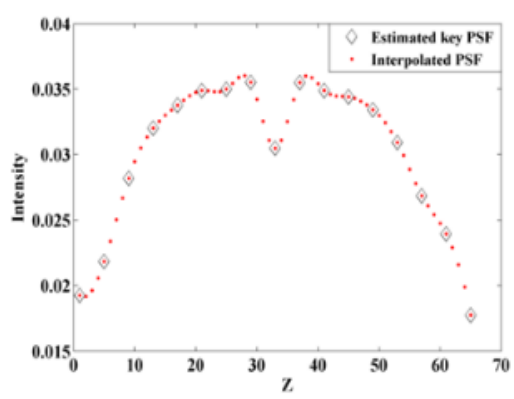

(a)

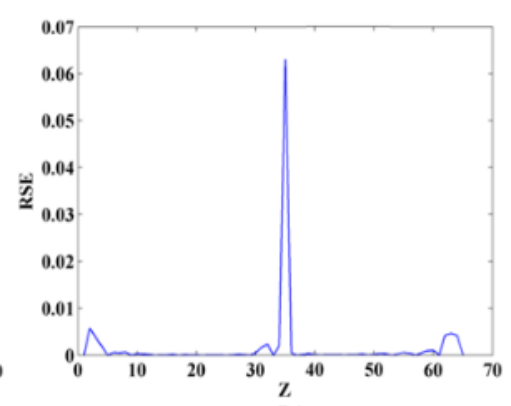

(b)

Fig.3. (a) The estimated and interpolated results, and (b) RSE between the estimated and interpolated PSFs. 
It can be seen from Fig. 3 (a) that cubic spline function can realize smoothing transition between two interpolation nodes. And Fig. 3 (b) shows that the interpolation error is satisfactory, although slight fluctuation occurs between $z=30$ and $z=40$. But the errors are less than 0.07 . And most of interpolation errors almost tend to 0 . The above results illustrates that space-variant PSFs can be generated using the proposed method instead of blind deconvolution method. Therefore we can significantly reduce calculation amount.

Fig. 4(a) and 4(b) are the 3D models reconstructed by RL-TV method [14] using SI-PSFs (, and variance ) and our method respectively, and both the iteration numbers are 10. For fairly comparing the efficiency of the methods, all results are the best experimental ones.

It can be seen from the Fig. 4 that the proposed method obtains the better result with less noise and clearer vision, compared with the method using SI-PSFs, especially in the edges of 3D fluorescence image model. Fig. 5 shows the SSIM values between original image stack and reconstructed image stack respectively using SI-PSF method (dotted line) and our method (solid line).

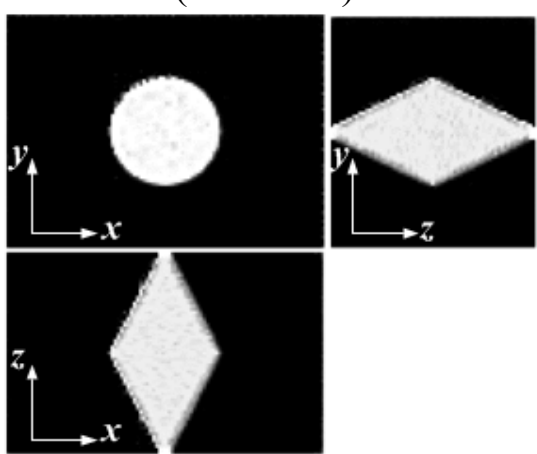

(a)

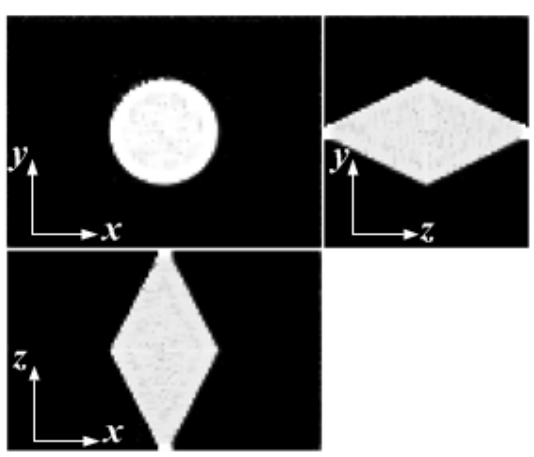

(b)

Fig.4. 3D models reconstructed by (a) SI-PSFs method, and (b) our method.

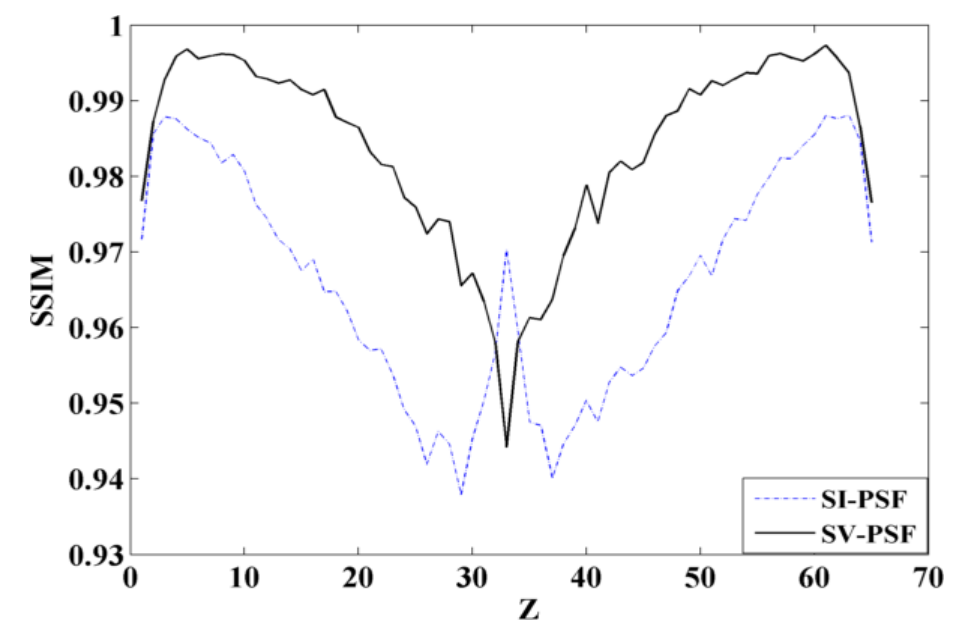

Fig.5. SSIM between original and reconstructed image stack respectively using SI-PSFs method (dotted line) and our method (solid line).

It can be seen from Fig. 5 that SSIM values obtained from our method are larger than ones from SI-PSFs method except some images between $\mathrm{z}=30$ and $\mathrm{z}=40$ along the optical axis. The reason is that the PSFs which are interpolated within this range might not be perfectly precise. This phenomenon can also be found in Fig. 3(b). But SSIMs between the original and the reconstructed image stack are still very large using our method, which exceed 0.94 . 


\section{Real Data}

We also do another experiment using real fluorescence image stack $(512 * 512 * 65)$ obtained from Internet and we don't know the type and any parameters of experimental instrument. Fig. 6(a) shows the intensities which are located in the same position of the estimated and interpolated PSFs. The errors between the estimated PSFs using blind deconvolution [9] and the interpolated PSFs using cubic spline theory are shown in Fig. 6(b).

It can be seen from Fig. 6 that both the interpolation results and interpolation errors are satisfactory. The fluctuation phenomenon of fitting error also occurs in Fig. 6 (b), but the RSE values are very small which do not exceed . Therefore cubic spline method can be adopted to generate more PSFs with the help of key PSFs. Fig. 7 (a)-(c) shows the 3D models of the original fluorescence microscopic image stack, the reconstructed image stacks by SI-PSFs method and our method respectively.

All the experimental results show that the reconstructed 3D model by SI-PSFs method looks more blurring, and false edges emerge in it. Our method, however, gives the clear result in the light of subjective human vision.

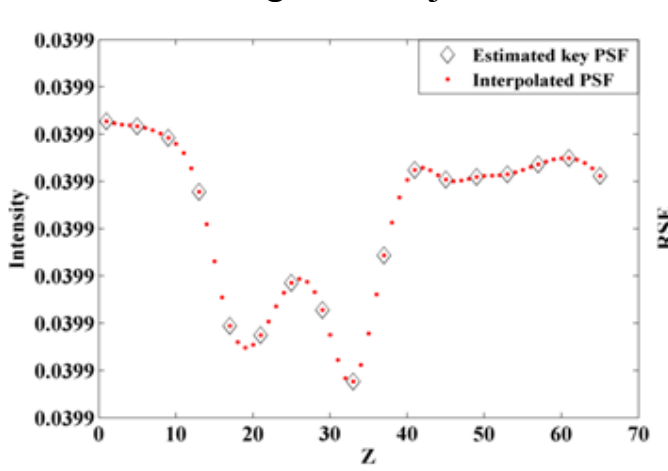

(a)

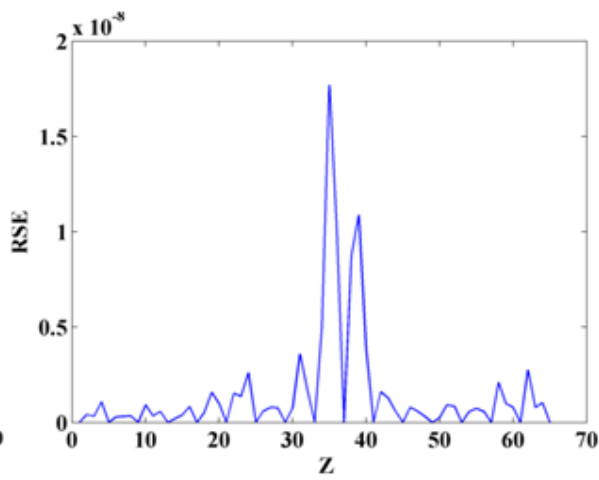

(b)

Fig.6. (a) The estimated and the interpolated results, and (b) RSE between the estimated and interpolated PSFs.

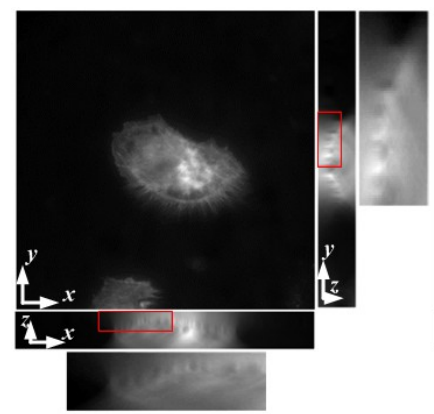

(a)

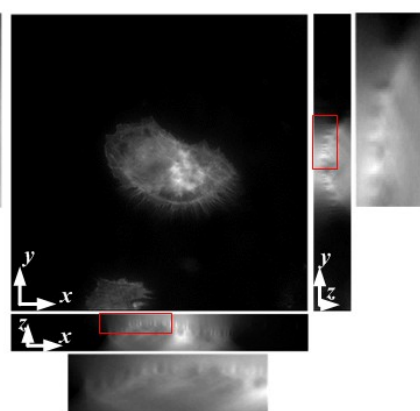

(b)

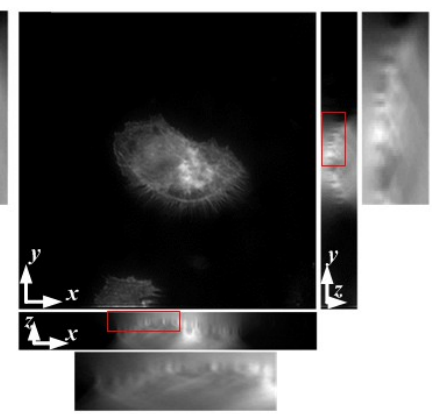

(c)

Fig.7. (a) 3D model of original image stack, and the reconstructed 3D models by (b) SI-PSFs method, and (c) our method.

\section{Conclusions}

A 3D reconstruction method of microscopic image is proposed in this paper. Firstly, key PSFs are estimated by blind deconvolution algorithm at several depths along the optical axis. Then, other PSFs are interpolated using cubic spline theory. Finally, the 3D microscopic specimen model can be reconstructed by this group of SV-PSFs. In 
addition, 3D Gaussian model is used to simulate the real PSF model, and SSIM and RSE are considered as measure criteria of the restored image stack and interpolated PSFs, respectively.

We applied the proposed method to the simulated and real data. The experimental results show that our method gives better restored image quality in the aspect of both the objective criterion and subjective vision. However, it should be noted that the estimation accuracy of the key PSFs has great influence to the interpolated PSFs and the final reconstructed results. Therefore, more accurate estimation algorithm for PSF is our future work.

\section{Acknowledgement}

This work is supported by Natural Science Foundation of China (No. 61671028), Beijing Natural Science Foundation (No. 4162018), Beijing Talents Fund (No. 201400 0026833ZK14), the Importation and Development of High-Caliber Talents Project of Beijing Munici-pal Institutions (No. CIT\&TCD201504010).

\section{References}

[1] N. Dey, L. Blanc-Féraud, C. Zimmer, P. Roux, Z. Kam, J. C. Olivo-Marin, and J. Zerubia, "3D microscopy deconvolution using Richardson-Lucy algorithm with total variation regularization," Research Report, RR-5272, INRIA, 2004.

[2] N. Dey, L. Blanc-Féraud, C. Zimmer, Z. Kam, J. C. Olivo-Marin, and J. Zerubia, "A deconvolution method for confocal microscopy with total variation regularization," IEEE International Symposium on Biomedical Imaging, pp. 1223-1226, Arlington, VA, USA, 15th-18th, Apr. 2004.

[3] S. B. Hadj, L. Blanc-Féraud, and G. Aubert, "Space variant blind image restoration,” Research Report, RR-8073, INRIA, 2012.

[4] S. Jia, J. C. Vaughan, and X. W. Zhuang, Journal of Nature photonics. 8(2014)302-306.

[5] S. Ghosh, and C. Preza, "Space-variant image formation for 3D fluorescence microscopy using a computationally efficient block-based model," IEEE 12th International Symposium on Biomedical Imaging, pp. 789-792, New York, USA, 16th-19th, Apr. 2015.

[6] S. B. Hadj, L. Blanc-Féraud, G. Aubert, and G. Englerl, "Blind restoration of confocal microscopy images in presence of a depth-variant blur and poisson noise," IEEE International Conference on Acoustics, Speech and Signal Processing, pp. 915-919, Vancouver, Canada, 26th -31st, May, 2013.

[7] J. Bardsley, S. Jefferies, J. Nagy, and R. Plemmons, Optics express. 14(2006)1767-1782.

[8] C. Preza, and J. A. Conchello, J. Opt. Soc. Am. A. 21( 2004)1593-1601.

[9] D. S. C. Biggs, and M. Andrews, Applied Optics, vol. 36(1997)1766-1775.

[10] L. B. Lucy, The Astronomical Journal. 79(1974)745-765. 
[11] C. Preza, M. I. Miller, L. J. Thomas, and J. G. McNally, J. Opt. Soc. Amer. A. 9(1992)219-228.

[12] P. J. Verveer, and T. M. Jovin, J. Opt. Soc. Amer. A. 14(1997)1696-1706.

[13]T. J. Holmes, J. Opt. Soc. Amer. A. 9(1992)1052-1061.

[14] N. Dey, L. Blanc-Feraud, C. Zimmer, P. Roux, Z. Kam, J. C. Olivo-Marin, and J. Zerubia, Microscopy research and technique. 69(2006)260-266.

[15] B. Zhang, J. Zerubia, and J. C. Olivo-Marin, Applied Optics. 46(2007)1819-1829.

[16] Z. Wang, A. C. Bovik, H. R. Sheikh, and E. P. Simoncelli, IEEE Transactions on Image Processing. 13(2004)600-612.

[17] Y. Wang, X. He, and H. Wang, "The Depth-Variant Image Restoration Based on Hopfield Neural Network," The 3rd International Conference on Natural Computation, pp.363-366, Haikou, China, 24th-27th, Aug. 2007. 